\title{
0 impacto da camptocormia na funcionalidade do idoso
}

\author{
Marlúcia de Fátima Rodrigues*, Célia Pereira Caldas**
}

\section{Resumo}

Durante o processo de envelhecimento muitas alterações ocorrem no organismo humano. A diminuição gradativa de massa muscular, da força e flexibilidade interfere na capacidade física e funcional do indivíduo. A camptocormia é descrita como uma postura anormal do tronco com severa flexão da coluna toracolombar e de joelhos que interfere na funcionalidade do idoso. Com o crescimento do número de idosos torna-se relevante o estudo das condições que podem diminuir a autonomia dessa população. Este estudo tem como objetivo descrever, com base na literatura científica nos campos da geriatria e fisioterapia, a camptocormia e como essa condição pode influenciar na funcionalidade do idoso. A etiologia da camptocormia não é bem compreendida. Há controvérsias entre os autores quanto às causas, pois tanto os fatores psíquicos quanto os orgânicos são reconhecidos como importantes. Uma constatação comum entre os pesquisadores é que uma postura excessivamente projetada para frente não pode ser considera- da normal. A desorganização imposta pela camptocormia nos sistemas ósseo, articular e muscular e a ausência de literatura nacional sobre o tema caracterizam a relevância do estudo. Além disso, é importante relacionar a camptocormia com alterações físicas e funcionais frequentemente encontradas em idosos. Para que fosse possível essa relação o estudo baseou-se na biomecânica das alterações posturais presentes na camptocormia, porém não relacionadas a ela na literatura. Estudos longitudinais posteriores são necessários, para que se possam descrever todas as alterações biomecânicas e funcionais, obter o diagnóstico cinético-funcional e uma forma de intervenção fisioterapêutica segura.

Palavras-chave: Idoso. Curvaturas da coluna vertebral. Doenças da coluna vertebral. Biomecânica. Envelhecimento. Modalidades de fisioterapia. Autonomia pessoal.

* Fisioterapeuta. Especialização em Geriatria e Gerontologia pela Universidade Estadual do Rio de Janeiro e em Anatomia e Biomecânica do Aparelho Locomotor pela Faculdade de Reabilitação da Associação de Solidariedade à Criança Excepcional. Discente do Grupo de Estudo de Epidemiologia da Universidade Aberta da Terceira Idade da Universidade Estadual do Rio de Janeiro. Endereço para correspondência: Estrada do Guerenguê, 1142, lote. 4, casa 4, CEP: 22.713-004, Taquara Rio de Janeiro - RJ. E-mail: malufisio1@gmail. com.br.

** Mestre em Saúde Coletiva do Instituto de Medicina Social da Universidade do Estado do Rio de Janeiro. Doutora em Enfermagem pela Universidade Federal do Rio de Janeiro. Pós-doutora em Gerontologia pela Universidade de Jönköping, Suécia. Professora adjunta da Universidade do Estado do Rio de Janeiro.

$\longrightarrow$ Recebido em março de 2009 - Avaliado em março de 2010.

$\hookrightarrow$ doi:10.5335/rbceh.2010.013 


\section{Introdução}

Compreender as inúmeras condições que podem interferir no movimento humano e na funcionalidade do idoso é um desafio para os cientistas. A autonomia do idoso é um tema muito estudado, em razão do número crescente desta população. Baseado nessa premissa, o estudo trata-se de uma revisão extensiva da literatura nacional e internacional nos campos da geriatria, gerontologia e fisioterapia, tendo como proposta descrever a camptocormia.

Segundo Micheli, Cersósimo e Piedmonte (2005), a camptocormia é uma condição caracterizada por severa flexão da coluna toracolombar e joelhos e curvatura passiva de ambos os braços, que aparece em posição ortostática e durante a marcha e desaparece em decúbito dorsal.

De acordo com Block et al. (2006), a camptocormia acomete principalmente indivíduos com mais de 65 anos; por isso, é atribuída ao envelhecimento e não é diagnosticada.

Para Rebelatto e Morelli (2004), acredita-se que o processo de envelhecimento seja progressivo e dinâmico e se caracteriza por alterações morfológicas, psicológicas, fisiológicas e bioquímicas que são determinantes em se tratando de vulnerabilidade e maior ou menor incidência de doenças e morte.

A camptocormia como origem, no sentido mais amplo, abrange um largo espectro de anormalidades desencadeadas. A severidade dos sintomas desenvolvidos detectados precocemente pode oferecer uma oportunidade de im- plementação de cuidados terapêuticos importantes antes de a morbidade se tornar significativa.

Dentre os inúmeros questionamentos que envolvem a camptocormia, sua origem tem despertado curiosidade. Não há consenso entre os autores quando se trata da etiologia da camptocormia. Essa questão foi intensamente discutida, porém ainda causa controvérsia, a saber, a influência dos fatores somáticos em oposição aos psíquicos.

Segundo Dias-Guzmán et al. (2006), embora a etiologia psicogênica tenha sido atribuída nos primeiros casos descritos, posteriormente foram considerados com mais frequência fatores neuromusculares e extrapiramidais. Portanto, os aspectos etiopatogênicos ainda são obscuros e com poucas experiências terapêuticas.

Para Norkin e Lavangie (2001), a postura ideal é obtida quando os seguimentos VER corporais estão alinhados de forma que os torques e as tensões sejam mínimos em toda a cadeia cinemática, onde as forças de compressão estão bem distribuídas sobre a superfície de descarga do peso corporal das articulações e nenhuma tensão excessiva é exercida sobre os ligamentos e músculos.

Segundo Magee (2002), na postura cifótica a flexão da cabeça e a protrusão do abdômen ocorrem para manter o centro de gravidade em sua posição normal. Por ser um transtorno que, por si só, pode desenvolver incapacidade funcional, limitações importantes e quadros álgicos severos em virtude da flexão anterior do tronco, essa alteração necessita de avaliação extensa das múltiplas 
causas que podem estar envolvidas na geração dos referidos problemas, buscando o conhecimento de sua evolução a fim de organizar diretrizes terapêuticas.

\section{Camptocormia}

O termo "camptocormia" deriva de duas palavras gregas: kamptos (curvar) e kormos (tronco). A curvatura patológica da coluna toracolombar foi descrita, primeiramente, pelo fisiologista e cirurgião Inglês Brodie em 1818. Brodie sugeriu que a dor lombar e a curvatura do tronco eram causadas por afecções orgânicas, processo destrutivo das vértebras e reações histéricas. (KARBOWSKI, 1999).

Segundo os mesmos autores, a primeira publicação em que o termo foi utilizado data de 1915, na revista Neurologique, em Paris, em trabalho de Souques e Rosanoff-Saloff. No texto os autores expunham breves considerações morfológicas sobre a curvatura patológica do tronco e explicavam que Souques propunha o termo "camptocormia" ( $\chi \alpha \mu \pi \tau \omega$, eu flexiono, eu curvo; $\chi о \rho \mu о \sigma$, o tronco), tendo em vista que até então havia diferentes nomes.

Segundo Azher e Jankovic (2005), a camptocormia é caracterizada por uma postura anormal do tronco, com severa flexão da coluna toracolombar, que aparece em posição ortostática e durante a marcha e desaparece em decúbito dorsal (DD).

Para Días-Guzmán et al. (2006), a camptocormia é uma perturbação de etiologia diversa. Embora a etiologia psicogênica fosse suposta em casos adiantados, diversos relatórios de causas orgânicas da camptocormia sugerem que a postura anormal pode resultar de lesões no estriado e no pálido (núcleo lenticular). (AZHER; JANKOVIC, 2005).

É sabido que o processo de envelhecimento gera algumas mudanças no organismo humano, porém não se pode atribuir a deformidades, um fator natural desse processo. Block et al. (2006) explicam que a camptocormia acomete principalmente indivíduos com mais de 65 anos, por isso é atribuída ao envelhecimento e não é diagnosticada. Para Slawek et al. (2006), embora uma ligeira postura encurvada seja uma característica do parkinsonismo, uma flexão extrema do tronco para frente não é comum. A origem da camptocormia é outro fator que merece atenção e deve ser investigada, visto que não há consenso entre os autores. Essa questão foi intensamente discutida, porém ainda causa controvérsias, a saber, a influência dos fatores somáticos em oposição aos psíquicos.

Segundo Dias-Guzmán et al. (2006), embora a etiologia psicogênica tenha sido atribuída nos primeiros casos descritos, posteriormente foram considerados com mais frequência fatores neuromusculares e extrapiramidais, em particular na doença de Parkinson (DP). Os aspectos etiopatogênicos ainda estão obscuros e existem poucos estudos sobre a terapêutica; recentemente, algumas pesquisas descreveram o parkinsonismo com camptocormia relacionado a processos inflamatórios focais do canal medular.

Slawek, Derejko e Mota (2003) descreveram a camptocormia como uma forma de distonia em DP e relatam que, 
embora a patogênese da camptocormia fosse diferente em varias desordens, em DP é ainda desconhecida. Entretanto, existe uma possibilidade de que um mecanismo central, envolvendo a degeneração do sistema dopaminérgico, seja responsável pela camptocormia. A melhora do quadro clínico doloroso e da flexão do tronco após palidotomia pode sugerir a natureza distônica da camptocormia em DP, fenômeno confirmado por Djaldetti e colaboradores (1999) e Friedman (2001), os quais sugeriram que a camptocormia em DP representava uma forma de distonia do tronco. Por sua vez, Sinel e Eisenberg, (1990) sugeriram que a etiologia da camptocormia seja multifatorial.

A camptocormia na DP poderia ser uma consequência da rigidez axial e fraqueza dos músculos eretores espinhais, possivelmente em vistude da disfunção dos gânglios basais em controlar a via reticuloespinhal. As principais projeções do percurso reticuloespinhal estão localizadas na musculatura axial e controlam a postura dinâmica e estática. Disfunção dos gânglios basais também poderia causar freezing e distúrbios autonômicos. $\mathrm{O}$ autor relata que esses distúrbios eram altamente prevalentes na população estudada. (LEPOUTRE, 2006).

Recentemente, a camptocormia, a distonia axial e o parkinsonismo foram relatados como uma expressão fenotípica da mutação do gene Parkin. A camptocormia também tem sido considerada como uma forma de distonia em doentes com DP, porém este conceito ainda causa controvérsias. (MICHELI; CERSÓSIMO; PIEDMONTE, 2005).
Para González (2002) a camptocormia é uma enfermidade de afecção muscular de aparição tardia, cuja sintomatologia consiste basicamente em lombalgia crônica, associada a uma cifose secundária e a debilidade da musculatura paravertebral lombar. A cifose é incrementada com a fadiga e representa uma relação mulher/homem de 4/1; em $20 \%$ dos casos se associa a um défice moderado da musculatura escapular e pélvica.

Sandoval (2001) descreveu a flexão do tronco como o principal sintoma da camptocormia. A marcha é dita antropoide, em razão do aspecto horizontal do dorso, os braços permanecem pendentes com balanceio símio e os joelhos apresentam uma semiflexão. A hiperextensão do pescoço produz uma hiperextensão cervical compensatória, que permite um olhar horizontal. A dor lombar tem sido descrita tanto nas formas miopáticas quanto nas psiquiátricas, de intensidade leve a moderada fundamentalmente no início do quadro e de forma transitória.

Segundo Slawek (2006), a camptocormia, a síndrome de Pisa e a antecollis podem representar a continuidade do mesmo fenômeno motor, e a maioria dos autores as relaciona a uma forma incomum de distonia axial em razão de muitas apresentações clinicas sobre diferentes formas de camptocormia/síndrome de Pisa. Os autores explicam que não é a etiologia, mas, sim, a localização da lesão específica, provavelmente dentro do putâmen, que é responsável por essa forma de distonia.

Lepoutre (2006) descreveu que a depleção dopaminergica presente em 
parkinsonianos pode contribuir para o desenvolvimento da camptocormia, pois provoca alterações funcionais na organização dos tratos corticoespinhal e retículoespinhal, que podem contribuir para a rigidez axial e a subutilização dos músculos paraespinhais, tornando-os progressivamente hipotrofiados.

Diversas causas clínicas, psíquicas e biomecânicas podem contribuir para $o$ desenvolvimento da camptocormia; por isso, novas pesquisas são necessárias a fim de obter diagnóstico preciso e terapêutica adequada.

O único estudo que traz uma visão biomecânica com análise cinemática, cinética e uma eletromiografia dinâmica é o de Hussein e Casey (1996). O autor declara que, embora essa incapacidade tenha sido previamente atribuída a uma desordem de conversão, pode haver um componente biomecânico. No estudo é descrito o caso de um homem de 47 anos que desenvolveu dor lombar, seguida por uma sensação de que para caminhar confortavelmente deveria assumir uma postura curvada para frente. Dois meses depois, embora a dor lombar estivesse resolvida, ele manteve o mesmo padrão de curvatura durante a marcha, provavelmente pelas contraturas adquiridas. $\mathrm{O}$ início da marcha era em posição ereta, porém após uns poucos passos ele inclinava espontaneamente o tronco. A análise mostrou uma excessiva inclinação pélvica anterior durante a fase terminal da marcha ereta e menor na curvada para frente. Segundo os autores, as razões prováveis para essa anormalidade são as contraturas encontradas nos músculos flexores do quadril bilate- ralmente e, talvez, a dor fosse aliviada assumindo a postura camptocórmica, pelo reduzido movimento de inclinação pélvica anterior.

O estudo demonstrou uma base biomecânica subjacente para a deficiência, que foi diagnosticada pela fisioterapia e tratada com sucesso. Os autores concluem que outros pacientes com camptocormia se beneficiariam de um exame físico detalhado e uma análise laboratorial do andar.

Van Gerpen (2006), em sua pesquisa, em que denominou "camptocormia distônica dopa-responsiva", descreveu o caso de um homem de 45 anos de idade que percebeu que, ao final do dia, particularmente após esforço físico, suas costas tendiam a se curvar. A curvatura podia ser aliviada momentaneamente se o paciente balançasse e estendesse seu tronco, entretanto em questão de segundos se curvava novamente e não conseguia andar ereto. O paciente respondeu bem ao tratamento com levodopa e nas raras ocasiões em que deixou de utilizar o medicamento houve recorrência da camptocormia. Em contrapartida, outros estudos

mostram que pacientes com distonia ou doença de Parkinson e camptocormia tratados nos últimos 25 anos com levodopa não obtiveram melhora da flexão do tronco durante a marcha.

Delcey et al. (2001) estudaram sete pacientes com camptocormia, cinco mulheres e dois homens, cuja idade variava entre 55 a 72 anos; todos tinham em comum uma miopatia axial visível na IRM. O autor descreve que a camptocormia muscular aparece como um 
sintoma que pode revelar uma miopatia axial em razão de múltiplas e variadas patologias. Assim, a descoberta de sua etiologia requer atenção, a fim de propor um tratamento adequado, que deveria ser associado à fisioterapia.

Lepoutre (2006) apresentou um estudo com 23 pacientes com idade média de $68,6(7,4)$ e o tempo médio do início dos sintomas da DP de 10,3 (5.1) anos; a duração da camptocormia era de 4,6 $(2,6)$ anos; 18 pacientes desenvolveram a flexão anterior do tronco num período superior a um ano; quatro pacientes relataram o aparecimento da curvatura em um tempo inferior a um ano; em um paciente $o$ aparecimento foi agudo (overnight). A camptocormia flutuava com $o$ tratamento dopaminérgico em cinco pacientes, com melhoria em todos os cinco. A extensão da postura curvada variava entre o ciclo diurno e era agravada com a fadiga e o estresse. Não houve correlação entre a duração da camptocormia e a duração do tratamento com L-dopa. A camptocormia determinada clinicamente variou de $40^{\circ}$, nos casos mais leves, até $90^{\circ}$, nos mais graves. Todos os pacientes $(\mathrm{n}=15)$ exibiram o mesmo padrão clínico de distúrbios posturais, com anteroversão da pelve (9/15), hipertonia fixa em flexão dos quadris (10/15), de joelhos $(15 / 15)$ e tornozelos (15/15) e paresia dos músculos paraespinhais (15/15).

Como descrito por Ozer et al. (2007), a miopatia axial é uma das causas mais frequentes de camptocormia. Os autores relataram o caso de um homem de 77 anos com diagnóstico de artrite reumatoide, aumento da curvatura do tronco que desaparecia em decúbito dor- sal, diagnosticada como camptocormia. Apresentava também sinais de parkinsonismo; a biópsia dos músculos paravertebrais revelou miopatia. A biópsia do bíceps sem alteração revela, segundo os autores, que apenas os músculos paravertebrais são afetados pela camptocormia. $\mathrm{O}$ paciente não respondeu ao tratamento com levodopa, por isso sendo descartada distonia axial ou DP. Embora a DP seja descrita em muitos pacientes com camptocormia, a miopatia deve ser sempre uma causa de suspeita, especialmente se o paciente não responde ao tratamento com levodopa.

Yamada et al. (2006) apresentaram uma paciente de 71 anos, com 11 anos de história de DP e cinco anos de camptocormia e que há poucos meses havia desenvolvido alucinações droga-indizida. A redução das drogas antiparkinsoniana aboliu sua psicose, porém exacerbou os sintomas motores (bradicinesia, perturbações da marcha e aumento da flexão anterior do tronco). A paciente foi submetida à estimulação bilateral do núcleo subtalâmico (ENST), em detrimento da estimulação do globo pálido interno (EGPi), visto que a diminuição das drogas antiparkinsonianas só poderia ser feita após a ENST. A estimulação produziu alívio da camptocormia. Os autores relataram que em dois estudos anteriores a ENST não produziu efeito. $\mathrm{O}$ contraste entre os resultados pode sugerir que pacientes com DP associada a camptocormia apresentam respostas diferentes a ENST, como no caso da terapêutica com levodopa.

A camptocormia é discutida por muitos autores no âmbito internacional, 
como também são descritas e discutidas sua etiologia e formas possíveis de tratamento clínico, porém em apenas um artigo foram descritas as alterações biomecânicas, deixando claro que o diagnóstico cinético-funcional e os métodos de intervenção fisioterapêuticas devem ser pesquisados.

\section{Impacto da camptocormia na funcionalidade do idoso}

De acordo com Pereira et al. (2006), dependência funcional pode ser definida como "a incapacidade de funcionar satisfatoriamente sem ajuda". A curvatura anterior do tronco altera a localização do centro de gravidade, que se torna mais anterior e afastado dos eixos articulares. Como a curvatura é mantida, as alterações podem se tornar fixas, modificando o alinhamento vertical; a compressão sobre as articulações é aumentada, gerando maior atividade muscular e tensão ligamentar. $\mathrm{O}$ conjunto desses fatores pode interferir diretamente no ortostatismo, no equilíbrio, na marcha e nas atividades da vida diária (AVDs).

Quanto maior for a distância entre o eixo articular e a linha da gravidade, maior será a magnitude do momento de força gravitacional, ou seja, a direção do momento de força gravitacional depende da localização da linha da gravidade, relativa a cada eixo articular. (NORKIN; LAVANGIE, 2001).

De acordo com Guccione (2002), a postura é o espelho do indivíduo; é a exteriorização do bem-estar ou sua ausência; é a representação da autoestima, da doença ou das vicissitudes da vida demonstradas no processo de envelhecimento. Em uma postura ideal, os seguimentos corporais devem estar alinhados de forma que os torques e as tensões sejam mínimos em toda a cadeia cinemática. (NORKIN; LEVANGIE, 2001).

O desalinhamento postural é um problema relevante e prevalente entre os idosos. A utilização excessiva de algumas articulações em detrimento de outras pode causar sérios problemas posturais, visto que as articulações devem ser utilizadas em conjunto para que as cargas sejam minimizadas.

Em uma postura ereta na coluna estática, um fio de prumo, análogo ao centro de gravidade, passa no meato externo do ouvido, atravessa o processo odontoide do áxis (C2) e atravessa os corpos de T1 e T2, através do promontório sacral; segue levemente posterior ao centro da articulação do quadril, anterior à articulação do joelho, pela articulação calcaneocuboidea do pé e ainda passa levemente anteriormente aos maléolos laterais. (CAILLIET, 2000).

$\mathrm{Na}$ camptocormia a linha da gravidade passa anteriormente aos eixos articulares, com o que o torque gera um movimento anterior, denominado "momento flexor". Como não ocorre movimento em direção oposta, os seguimentos corporais são alterados.

Cailliet (2003) explica que, quando a cifose torácica está aumentada, a cabeça se encontra à frente do centro de gravidade. A cabeça de um adulto pesa, em média, de 4 a $6 \mathrm{~kg}$. Se considerarmos a cabeça pesando $5 \mathrm{~kg}$ e se for mantida a $7,5 \mathrm{~cm}$ à frente do centro de gravidade, a cabeça passa a pesar $15 \mathrm{~kg}$, com o que as 
consequências posturais são inúmeras, pois, além do estiramento do pescoço, que pode causar sequelas importantes, há alterações somáticas que causam incapacidade e desconforto.

Para o mesmo autor, o indivíduo que mantém uma postura com a cabeça para frente pode diminuir a capacidade de virar a cabeça entre $25 \%$ e $50 \%$ para a direita e/ou para a esquerda pela diminuição do $\mathrm{ADM}$ rotacional ativa e passivamente, com o agravo de se tornar patológico se mantida persistentemente. As articulações são muito exigidas e ficam expostas a traumas recorrentes. A lordose cervical aumentada fecha os forames intervertebrais comprimindo as raízes nervosas; as cápsulas articulares tendem a aderir, ocasionando limitação estrutural gradativa; com as mudanças na estrutura da cartilagem, as articulações facetarias degeneram, causando artrite. Se houver tensão muscular superposta, a compressão é aumentada.

Um estudo que teve como objetivo analisar o perfil postural de idosos realizado por Pastre et al. (2004) mostrou que $75 \%$ da população estudada apresentava aumento da cifose torácica. De acordo com Cailliet (2000), os locais de formação de osteófitos são aqueles que apresentam pontos de maior pressão; logo, são aqueles de maior concavidade e mais distantes da linha de gravidade.

$\mathrm{Na}$ flexão do joelho em $15^{\circ}$, o músculo quadríceps aumenta sua atividade para manter a postura ereta de zero para $22^{\circ}$. Com o joelho em $30^{\circ}$ de flexão, a atividade do quadríceps aumenta para $51 \%$; por consequência, as articulações tibiofemoral e patelofemoral estão sujeitas a um estresse compressivo maior que o normal. (NORKIN; LAVANGIE, 2001).

Segundo Kapandji (2003), a flexão do joelho é o ponto máximo de instabilidade para suas articulações, quando está sujeito ao máximo de lesões meniscais e ligamentares. Cristopoliski et al. (2008) relatam que a flexão do joelho influencia na altura mínima do metatarso em relação ao solo durante a fase de balanço médio, interferindo na marcha.

Norkin e Levangie (2001) descreveram a ação de flexão do joelho e relataram que cinco dos sete músculos que fletem o joelho são biarticulares (cruzando o quadril como extensores e o joelho como flexores). Dessa forma, sua habilidade em produzir força efetiva pode ser influenciada pelo posicionamento das duas articulações.

Outras alterações biomecânicas importantes são encontradas no aumento mantido da curvatura dorsal: os ombros descem e rodam internamente, de modo que as escápulas rodam para baixo, as costelas descem e afundam, tendo como consequência a diminuição da expansão pulmonar. (CAILLIET, 2003).

Pickles et al. (1998) descrevem o aumento da cifose torácica como a mais frequente anomalia postural presente em idosos e que interfere diretamente na lordose lombar como medida compensatória; modifica também as costelas, aumentando seu diâmetro posterior, interferindo nos movimentos da escápula e em toda musculatura envolvida direta ou indiretamente com essas estruturas.

A elevação do braço de até 120 é possível pelo posicionamento em rotação externa (RE), posição que permite 
que o tubérculo maior passe por trás do acrômio. Quando se assume uma postura cifótica dorsal com os ombros arredondados internamente, o braço se encontra em rotação interna (RI) e perde $30^{\circ}$ em abdução, elevando-se somente até $90^{\circ}$. A escápula modifica seu alinhamento vertical e roda para frente e para baixo, deprimindo o processo acromial e alterando a posição da fossa glenoide. Esse posicionamento não permite que os braços se elevem acima da cabeça. A relação acrômio e cabeça do úmero abduzida altera-se, ocorre a intrusão do tubérculo maior e de todos os tecidos contidos no compartimento supraumeral em todas as atividades do braço que exijam elevação e abdução acentuadas. Uma possibilidade constante é o pinçamento do manguito rotador. (CAILLIET, 2000).

A camptocormia é uma condição altamente incapacitante, caracterizada por um progressivo enfraquecimento da musculatura paravertebral, que afeta, predominantemente, a população idosa. É associada a condições musculoesqueléticas e neurológicas, incluindo as distrofias musculares, miopatias inflamatórias, distonia segmentar, esclerose lateral amiotrófica, DP, camptocormia idiopática, entre outras. Um diagnóstico precoce é necessário para iniciar o tratamento adequado e evitar a progressão das deformidades espinhais. (GÓMEZPUERTA et al., 2006).

A camptocormia em DP é a mais provável manifestação num grupo de desordens heterogêneas; tanto nos casos de DP como nos de parkinsonismo as atenções têm sido centradas em envolvimento neuromuscular. Pode levar a uma multiplicidade de fatores que interferem diretamente na autonomia e funcionalidade de idosos, como, por exemplo, desordens osteomioarticulares, dor e distúrbio de equilíbrio e marcha.

Uma análise biomecânica detalhada da camptocormia pode encontrar muitas mudanças posturais em idosos, as quais podem causar dor, desconforto e diminuição da capacidade funcional.

\section{Considerações finais}

Mudanças na pirâmide etária em curso no Brasil exigem uma nova demanda de atitudes a fim de identificar, qualificar e intervir nos aspectos que influenciam na manutenção da capacidade funcional e na autonomia do indivíduo idoso.

Concomitantemente à transição epidemiológica, com predominância das patologias crônicas, prevalecem as alterações posturais como desordens comuns e multifatoriais. Fatores psíquicos, como a dificuldade de lidar com a perda de entes queridos e problemas familiares, ou orgânicos, como os problemas neurológicos ou osteomioarticulares, podem culminar com alterações posturais.

A camptocormia, apesar de sua prevalência e das implicações negativas sobre a funcionalidade de idosos, ainda é pouco estudada. Detectar as alterações precocemente pode evitar que uma cascata de desvios seja instalada, sobrecarregando outros segmentos corporais, modificando os padrões de força, agilidade e controle postural, tendo como consequência deficiência de marcha e de equilíbrio, tornando o indivíduo frágil e, portanto, vulnerável a quedas.

RBCEH, Passo Fundo, v. 7, n. 1, p. 132-143, jan./abr. 2010 
A referência etiológica da camptocormia é muito importante e está sendo investigada, como também o estabelecimento de intervenção clínica. Portanto, faz-se necessária a investigação dos parâmetros biomecânicos. Analisar a camptocormia por uma visão biomecânica, destacando os desvios posturais, estáticos e dinâmicos e o quanto esses influenciam na qualidade de vida dos idosos, bem como em suas AVDs, pode estabelecer a distância entre o sucesso e o insucesso da intervenção fisioterapêutica nos declínios de equilíbrio e marcha.

Entretanto, as ações não devem se limitar a idosos que já apresentam camptocormia. É importante salientar que estudos voltados para a prevenção primária dessa anomalia, com a prescrição de atividades físicas que preconizem o alongamento, o fortalecimento das cadeias musculares e de músculos intrínsecos e orientações para a manutenção de posturas simétricas, mostram-se relevantes.

Por meio desse estudo percebeu-se que há deficiência literária na investigação da camptocormia no contexto cinético-funcional, porém essa deficiência precisa ser corrigida. É fato que as alterações físicas impostas ao idoso pela camptocormia comprometem suas atitudes funcionais no âmbito da realização de suas AVDs e é possível afirmar que as mudanças posturais influenciam diretamente nas atitudes comportamentais, restringindo o idoso a pequenas tarefas e impedindo-o, assim, de ter autonomia.

Em razão pouca literatura existente com base biomecânica, não foi possível relacionar e discutir as alterações con- sequentes da postura camptocórmica. Assim, o que se fez foi idebtificar as influências na funcionalidade do idoso das alterações posturais descritas e que também estão presentes na camptocormia.

A visão humanizada e holística de uma equipe multidisciplinar pode contribuir no processo preventivo da camptocormia, impedindo que as alterações avancem e imponham limitação funcional, transtorno de marcha e equilíbrio, interferência na imagem corporal, aumentando o medo de cair e o risco de quedas.

The impact of the camptocormia in the functionality of the aged one

\section{Abstract}

During the aging process many alterations occur in the human organism. The gradual reduction of muscular mass, force and flexibility intervenes with the physical and functional capacity of the individual. Camptocormia is described as an abnormal posture of the trunk with severe bending of the thoracolumbar column and knees that intervenes with the functionality of the aged one. With the increasing number of aged people the study of the conditions which can diminish the autonomy of this population becomes relevant. The aim of this study is to describe, from a scientific ground on Geriatrics and Physiotherapy literature, both camptocormia and how this condition can influence the functionality of the aged one. The etiology of camptocormia is not well understood as yet. Authors show controversy as to its causes. Both psychic and organic factors are recognized as important. It is commonly established among researchers that an excessively forward projected posture cannot 
be considered normal. The disorganization imposed by camptocormia to the bones, articulations and muscular systems and the lack of Brazilian literature on the subject make for the study relevance. Moreover, it is important to investigate the relation of camptocormia to physical and functional alterations frequently found in aged people. To make it possible the study was based on the biomechanics of postural alterations shown in camptocormia, however unrelated to it in scientific literature. Further studies on longitudinal body posture are necessary, so that all the biomechanical and functional alterations can be described and the kinetic-functional diagnosis obtained, as well as a safe form of physiotherapeutic intervention is found.

Key words: Elderly. Spinal curvatures. Spinal diseases. Biomechanics. Aging. Physical therapy modalities. Personal autonomy.

\section{Referências}

AZHER, S. N.; JANKOVIC, J. Camptocormia, pathogenesis, classification, and response to therapy. Neurology, v. 65, n. 3, p. 355-359, 2005.

BLOCK, F. et al. Parkinson's disease with camptocormia. J. Neurol. Neurosurg. Psychiatry, v. 77, s. n, p. 1223-1228, June 2006.

CAILLIET, RENE. Dor cervical e no braço. 3. ed. Porto Alegre: Artmed, 2003.

Dor no ombro. 3. ed. Porto Alegre: Artmed, 2000.

CRISTOPOLISKI, F. et al. Efeito transiente de exercícios de flexibilidade na articulação do quadril sobre a marcha de idosas. Revista Brasileira de Medicina do Esporte, São Paulo, v. 14, n. 2, p. 139-144, mar./abr. 2008.

DELCEY, V. et al. La camptocormie: um signe de myopathie axiale, à propos de sept observations. Rev. Méd. Interne, v. 23, n. 2, p. 144-154, Feb. 2002.
DÍAS-GUZMÁN, J. et al. Parkinsonismo y camptocormia con miopatia focal espinal: descripción de un caso y respuesta al tratamiento. Revista de Neurologia, v. 43, n. 8, p. 466-469, 2006.

GÓMEZ-PUERTA, J. A. Camptocormia as a clinical manifestation of mitochondrial myopathy. Clinical Rheumatol, v. 26, s. n, p. 1017-1019, 2007.

GONZÁLEZ, M. M. J. Camptocormia: uma enfermedad muscular infrecuente. An. Med. Interna, v. 19, n. 9, p. 470-472, 2002.

GUCCIONE, A. A. Fisioterapia geriátrica. 2. ed. Rio de Janeiro: Guanabara Koogan, 2002.

HUSSEIN, A.; CASEY, K. Camptocormia: A biomechanical analysis: a case report. American Journal of Psysical Medicine \& Rehabilitation. v. 75, n. 4, p. 310-313, Jul./ Aug. 1996.

KAPANDJI, A. I. Fisiologia articular: tronco e coluna vertebral. Rio de Janeiro: Manole, 2000.

KARBOWSKI, K. The old and the new camptocormia. Spine, v. 24, n. 14, p. 1494-1498, 1999.

LEPOUTRE, A. C. et al. A specific clinical pattern of camptocormia in Parkinson's disease. J. Neurol. Neurosurg. Psychiatry., v. 77, n. 11, p. 1229-1234, Nov. 2006.

MAGGE, D. J. Avaliação musculoesquelética. São Paulo: Manole, 2002.

MICHELI, F.; CERSÓSIMO, M. G.; PIEDIMONTE, F. Camptocormia in a patient with Parkinson disease: beneficial effects of pallidal deep brain stimulation. Case report. $J$. Neurosurg., v. 103, n. 6, p. 1081-1083, Dec. 2005.

NORKIN, C. C.; LEVANGIE, P. K. Articulações estrutura e função: uma abordagem prática e abrangente. 2. ed. Rio de Janeiro: Revinter, 2001.

OZER, F. et al. Camptocormia in a patient with Parkinson disease and a myopathy with nemaline rods. American Journal Phys. Med. Rehabil, v. 86, n. 1, p. 3-6, Jan. 2007. 
PASTRE, C. M. Alterações posturais no atletismo: estudo analítico da seleção brasileira nos Jogos Pan-Americanos de Santo Domingo 2003. Revista Fisioterapia Brasil, São Paulo, v. 5, n. 6, p. 457-461, nov./dez. 2004.

PEREIRA, L. S. M. et al. Adaptação transcultural e análise da confiabilidade do Southampton Assessment of a Mobility para avaliar a mobilidade de idosos brasileiros com demência. Cadernos de Saúde Pública, Rio de Janeiro, v. 22, n. 10, p. 2085-2095, out. 2006.

PICKLES, B. et al. Fisioterapia na terceira idade. São Paulo: Santos, 1998.

REBELATT0, J. R.; MORELLI, J. G. S. Fisioterapia geriátrica: a prática da assistência ao idoso. São Paulo: Manole, 2004.

SANDOVAL, P. Redescubriendo la camptocormia. Escuela de Medicina de Pontificia Universidad Católica de Chile, 2001. Disponível em: <escuela.med.puc.cl/cuadernos/2001/12.html>. Acesso em: 5 mar. 2007.

SLAWEK, J. et al. Camptocormia or Pisa syndrome in multiple system atrophy. Clinical Neurology and Neurosurgery, v. 108, n. 7, p. 699-704, 2006.

SLAWEK, J.; DEREJKO, M.; LASS, P. Camptocormia as a form of dystonia in Parkinson's disease. European Journal of Neurology, v. 10, n. 1, p.107-110, 2003.

VAN GERPEN, J. A. Dopa-responsive dystinic camptocormia. Rev. Neurology., v. 66, n. 11, 2006.

YAMADA, K. Alleviation of camptocormia by bilateral subthalamic nucleus stimulation in a patient with Parkinson's disease. Parkinsonism and Related Disorders, v. 12, n. 6, p. 372-375, 2006. 